\title{
Pleiotrophin-induced endothelial cell migration is regulated by xanthine oxidase-mediated generation of reactive oxygen species
}

\author{
Sotiria Tsirmoula ${ }^{\mathrm{a}}$, Margarita Lamprou ${ }^{\mathrm{a}}$, Maria Hatziapostolou ${ }^{\mathrm{a}, 1}$, Nelly Kieffer $^{\mathrm{b}}$, Evangelia Papadimitriou ${ }^{\mathrm{a}, *}$ \\ a Laboratory of Molecular Pharmacology, Department of Pharmacy, University of Patras, GR26504 Patras, Greece \\ b Sino-French Research Centre for Life Sciences and Genomics, CNRS/LIA124, Rui Jin Hospital, Jiao Tong University Medical School, 197 Rui Jin Er Road, Shanghai 200025, China
}

\section{A R T I C L E I N F O}

\section{Article history:}

Accepted 3 January 2015

Available online 10 January 2015

\section{Keywords:}

Cell migration

PTN

ROS

$\mathrm{RPTP} \beta / \zeta$

Xanthine oxidase

$\alpha \beta_{3}$

\begin{abstract}
A B S T R A C T
Pleiotrophin (PTN) is a heparin-binding growth factor that induces cell migration through binding to its receptor protein tyrosine phosphatase beta/zeta (RPTP $\beta / \zeta)$ and integrin alpha v beta $3\left(\alpha_{v} \beta_{3}\right)$. In the present work, we studied the effect of PTN on the generation of reactive oxygen species (ROS) in human endothelial cells and the involvement of ROS in PTN-induced cell migration. Exogenous PTN significantly increased ROS levels in a concentration and time-dependent manner in both human endothelial and prostate cancer cells, while knockdown of endogenous PTN expression in prostate cancer cells significantly down-regulated ROS production. Suppression of RPTP $\beta / \zeta$ through genetic and pharmacological approaches, or inhibition of c-src kinase activity abolished PTN-induced ROS generation. A synthetic peptide that blocks PTN $-\alpha_{\nu} \beta_{3}$ interaction abolished PTNinduced ROS generation, suggesting that $\alpha_{v} \beta_{3}$ is also involved. The latter was confirmed in CHO cells that do not express $\beta_{3}$ or over-express wild-type $\beta_{3}$ or mutant $\beta_{3}$ Y773F/Y785F. PTN increased ROS generation in cells expressing wild-type $\beta_{3}$ but not in cells not expressing or expressing mutant $\beta_{3}$. Phosphoinositide 3-kinase (PI3K) or Erk1/2 inhibition suppressed PTN-induced ROS production, suggesting that ROS production lays down-stream of PI3K or Erk1/2 activation by PTN. Finally, ROS scavenging and xanthine oxidase inhibition completely abolished both PTN-induced ROS generation and cell migration, while NADPH oxidase inhibition had no effect. Collectively, these data suggest that xanthine oxidase-mediated ROS production is required for PTN-induced cell migration through the cell membrane functional complex of $\alpha_{v} \beta_{3}$ and RPTP $\beta / \zeta$ and activation of c-src, PI3K and ERK1/2 kinases.
\end{abstract}

(c) 2015 Elsevier Inc. All rights reserved.

\section{Introduction}

Pleiotrophin (PTN) is a heparin binding growth factor with a significant regulatory role on inflammation, angiogenesis and cancer (Pantazaka and Papadimitriou, 2012; Papadimitriou et al., 2009; Silver et al., 2012; Yokoi et al., 2012; Zhang et al., 2013). In all cases, the best characterized PTN function is its effect on cell migration through its receptor protein tyrosine phosphatase beta/zeta (RPTP $/ / \zeta$ ) (Lu et al., 2005; Polykratis et al., 2005) and integrin alpha v beta $3\left(\alpha_{\nu} \beta_{3}\right)$ (Mikelis et al., 2009). Part of the downstream signaling has been elucidated and involves c-Src kinase, phosphoinositide 3-kinase (PI3K) and ERK1/2 activation (Koutsioumpa et al., 2013; Mikelis et al., 2009; Polykratis et al., 2005). However, a lot of work is still required in order to uncover more players, as well as to pinpoint how all these players communicate to regulate the effect of PTN on cell migration.

\footnotetext{
* Corresponding author.

E-mail address: epapad@upatras.gr (E. Papadimitriou).

1 Current address: Center for Systems Biomedicine, Division of Digestive Diseases, David Geffen School of Medicine, University of California Los Angeles, Los Angeles, CA, USA.
}

Reactive oxygen species (ROS), like hydrogen peroxide, superoxide and the hydroxyl radical, are constantly produced during normal metabolism and in response to external stimuli. At higher concentrations following oxidative stress situations, ROS may damage proteins, lipids and DNA, and may induce apoptosis. In contrast, after stimulation by various external factors, including growth factors, hormones and cytokines, or cellular events such as adhesion, cells will produce low local amounts of ROS (Dhar-Mascareno et al., 2003; Hatziapostolou et al., 2006; Terman et al., 1992), which have been identified as essential mediators that regulate the transduction of signals from the membrane to the nucleus via oxidation and reduction in proteins (Holmström and Finkel, 2014) and lead to angiogenesis stimulation (Kim and Byzova, 2014; Polytarchou and Papadimitriou, 2004, 2005, Polytarchou et al., 2009) and inflammation linked to several pathologies (Bryan et al., 2012; Naik and Dixit, 2011). ROS are produced by a number of pathways, including the mitochondrial electron transport chain, cytochrome $\mathrm{P} 450$, xanthine oxidase (XO), uncoupled nitric oxide synthase and the family of NADPH oxidase enzymes (Bir et al., 2012; Wilkinson-Berka et al., 2013).

The aim of the present study was to investigate whether PTN affects ROS production by human endothelial and prostate cancer cells and whether ROS production is involved in PTN-induced cell migration. 


\section{Materials and methods}

\section{Materials}

Human recombinant PTN was from PeproTech, Inc. (Rocky Hill, NJ, USA). The NAD $(P) H$ oxidase-specific inhibitor 4-hydroxy-3methoxyacetophenone (apocynin) and the ROS-sensitive fluorescent dye, 5(6)-carboxy-2,7-dichlorodihydrofluorescein diacetate (carboxy- $\mathrm{H}_{2}$ DCFDA) were purchased from Fluka. The hydrogen peroxide scavenger catalase, the $\mathrm{NAD}(\mathrm{P}) \mathrm{H}$ oxidase inhibitors 4-(2aminoethyl)benzenesulfonylfluoride (AEBSF) and 3-benzyl-7-(2benzoxazolyl)thio-1,2,3-triazolo(4,5-d)pyrimidine (VAS2780), the XO inhibitor allopurinol and the general phosphatase inhibitor sodium orthovanadate were purchased from Sigma. The XO inhibitor febuxostat was from Santa Cruz Biotechnology, Inc. The mitogen-activated protein kinase/extracellular signal-regulated kinase kinase (MEK) inhibitor U0126 and the src kinase inhibitor PP1 were purchased from Tocris (Ellisville, MO, USA). RNA oligonucleotide primers for RPTP $\beta / \zeta$ (Polykratis et al., 2005) were obtained from VBCBiotech Services (Vienna, Austria), and for human XO were obtained from Santa Cruz Biotechnology, Inc. Double-stranded negative control siRNA was from Ambion (Austin, TX, USA). B3 (CYDMKTTC) and B3 scrambled (TKCMTCDY) peptides were from Cambridge Peptides (Birmingham, UK). All the inhibitors and antioxidants at the concentrations used were not toxic to the cells (data not shown).

\section{Cell culture}

Human umbilical vein endothelial cells (HUVEC), human glioma M059K cells, Chinese hamster ovary ( $\mathrm{CHO}$ ) cells deficient in endogenous $\beta_{3}$ and human prostate cancer epithelial cell lines LNCaP and PC3 were cultured as previously described (Hatziapostolou et al., 2005; Koutsioumpa et al., 2013; Tsirmoula et al., 2012). Stable CHO clones expressing wild-type $\beta_{3}$ or mutant $\beta_{3}$ Y773F/Y785F were generated as previously described (Mikelis et al., 2009). Cell culture reagents were from BiochromKG (Berlin, Germany). All cultures were maintained at $37{ }^{\circ} \mathrm{C}, 5 \% \mathrm{CO}_{2}$ and $100 \%$ humidity. When cells reached $80-90 \%$ confluence, they were serum-starved for $16 \mathrm{~h}$ before performing any further experiments.

\section{Migration assay}

Migration assays were performed as previously described (Koutsioumpa et al., 2013) in 24-well microchemotaxis chambers (Corning, Inc., Lowell, MA, USA) using uncoated polycarbonate membranes with 8 - $\mu \mathrm{m}$ pores. Serum-starved cells were harvested and resuspended at a concentration of $10^{5}$ cells $/ 0.1 \mathrm{ml}$ in serum-free medium containing $0.25 \%$ bovine serum albumin (BSA). The bottom chamber was filled with $0.6 \mathrm{ml}$ of serum-free medium containing $0.25 \%$ BSA and the tested substances. The upper chamber was loaded with $0.1 \mathrm{ml}$ of medium containing the cells and incubated for $4 \mathrm{~h}$ at $37^{\circ} \mathrm{C}$. After completion of the incubation, the filters were fixed and stained with $0.33 \%$ toluidine blue solution. The cells that migrated through the filter were quantified by counting the entire area of each filter, using a grid and an Optech microscope (Optech Microscope Services Ltd., Thame, UK) at an $\times 20$ objective.

\section{RNA interference}

HUVEC were grown to $50 \%$ confluence in medium without antibiotics. Transfection was performed in serum-free medium for $4 \mathrm{~h}$ using annealed RNA for RPTP $\beta / \zeta$ at the concentration of $50 \mathrm{nM}$ and jetSI-ENDO (Polyplus Transfection, Illkirch, France) as transfection reagent (Polykratis et al., 2005 ), or $X O$ at the concentration of $50 \mathrm{nM}$ and Lipofectamine ${ }^{\circledR}$ RNAiMAX transfection reagent (Life Technologies). Cells were incubated for another $48 \mathrm{~h}$ for $\mathrm{RPTP} \beta / \zeta$ and $72 \mathrm{~h}$ for $\mathrm{XO}$ in serum-containing medium and serum starved before further experiments. Doublestranded negative control siRNA was used in all experiments.

\section{Assay of intracellular ROS production}

ROS were assayed using the ROS-sensitive fluorescent dye carboxy- $\mathrm{H}_{2}$ DCFDA, as previously described (Hatziapostolou et al., 2006). Cells were seeded in 12 -well plates at a concentration of $2 \times 10^{5}$ cells/well. Twenty four hours after seeding, LNCaP and PC3 cells were directly assayed for carboxy-DCF fluorescence, as described below, while HUVEC, M059K and CHO cells were serumstarved and then incubated in serum-free medium containing 0.25\% BSA and PTN for the indicated periods of time. When used, antioxidants were added into the cell culture medium 30 min prior to PTN stimulation. At the end of the incubation period, cells were washed with phosphate-buffered saline (PBS) $\mathrm{pH} 7.4$, and then incubated in the dark for 15 min in Ham's F-12 lacking phenol red, containing $50 \mu \mathrm{M}$ carboxy- $\mathrm{H}_{2}$ DCFDA. Cells were released with trypsin/EDTA and lysed. Soluble extracts were prepared by centrifugation for removal of cell debris and fluorescence intensity was determined spectrophotometrically, using an excitation wavelength of $485 \mathrm{~nm}$ and emission wave-length of $500 \mathrm{~nm}$. Cell lysates were analyzed for protein content using the Bradford method, and carboxy-DCF fluorescence was normalized for total protein content.

\section{Statistical analysis}

The significance of variability between the results of each group and its corresponding control was determined by unpaired $t$ test. Each experiment included triplicate measurements for each condition tested, unless otherwise indicated. All results are expressed as mean \pm s.e.m. from at least three independent experiments.

\section{Results}

PTN increases carboxy-DCF fluorescence in a concentration- and time-dependent manner

Treatment of HUVEC with PTN at the concentration of $100 \mathrm{ng} / \mathrm{ml}$ that causes maximal stimulation of HUVEC migration (Polykratis et al., 2005) resulted in a time-dependent increase in carboxy-DCF fluorescence, which was maximal $10 \mathrm{~min}$ after PTN stimulation (Fig. 1A). Treatment of cells with different concentrations of PTN for $15 \mathrm{~min}$ resulted in a concentration-dependent increase in carboxy-DCF fluorescence (Fig. 1B).

Similarly to HUVEC, PTN increased carboxy-DCF fluorescence in a concentration- and time-dependent manner also in human prostate cancer LNCaP cells (Supplementary Figure S1). We have previously shown that down-regulation of endogenous PTN in human prostate cancer LNCaP (Hatziapostolou et al., 2005) or PC3 (Tsirmoula et al., 2012) cells results in decreased ability of the cells to migrate and form colonies in soft agar in vitro, increased apoptosis and decreased growth in immunocompromised rats in vivo. In the present study, we used the same cells stably expressing antisense PTN and investigated whether endogenous PTN levels correlated with intracellular carboxy-DCF fluorescence. As shown in Fig. 2, intracellular carboxy-DCF fluorescence was decreased in both LNCaP and PC 3 cells with decreased expression of endogenous PTN. In cells transfected with the appropriate control vector, carboxy-DCF fluorescence was similar to those in the corresponding non-transfected cells.

\section{$R P T P \beta / \zeta$ is involved in PTN-induced elevation of carboxy-DCF fluorescence}

Since RPTP $\beta / \zeta$ is one of the main PTN receptors, we studied whether $\mathrm{RPTP} \beta / \zeta$ is involved in PTN-induced carboxy-DCF fluorescence elevation. Treatment of HUVEC with the protein tyrosine phosphatase 
A

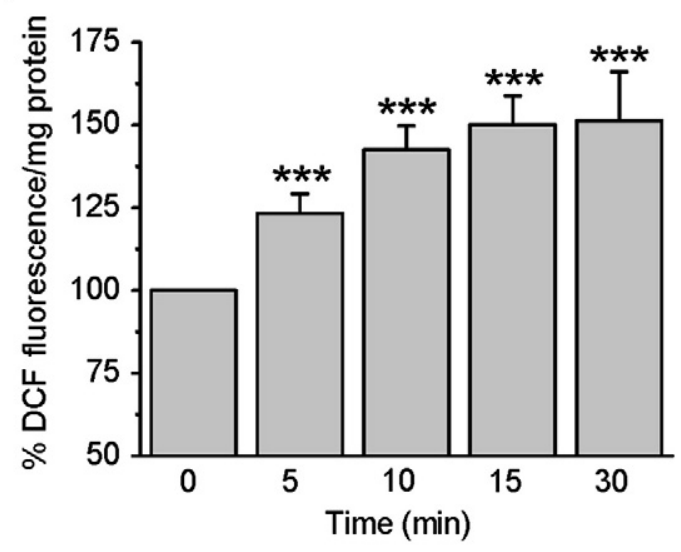

B

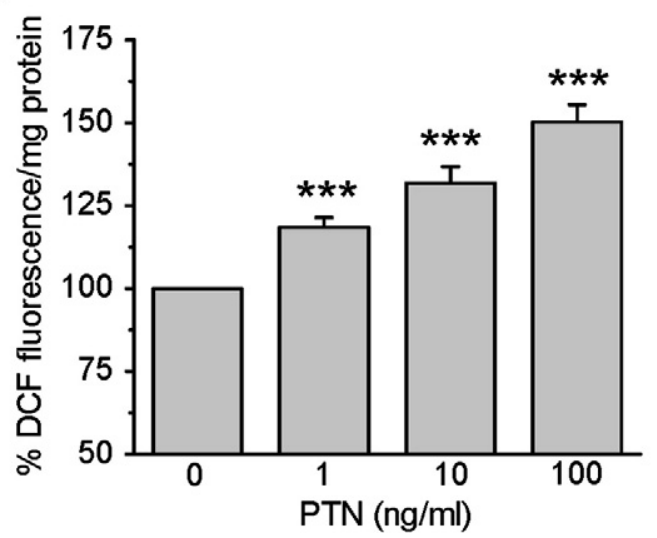

Fig. 1. PTN increases carboxy-DCF fluorescence in a dose- and time-dependent manner. Serum starved HUVEC were stimulated with $100 \mathrm{ng} / \mathrm{ml}$ PTN for different time points (A) or with different concentrations of PTN for $15 \mathrm{~min}$ (B). In all cases, the ratio carboxy-DCF fluorescence/mg of total protein was calculated for each sample. Results are expressed as mean \pm s.e.m. of the percent change of carboxy-DCF fluorescence per $\mathrm{mg}$ of total protein in PTN-stimulated cells compared with the untreated cells (set as default $=100$ ). Asterisks denote a statistically significant difference from untreated cells. ${ }^{* * *} P<0.001$

inhibitor sodium orthovanadate for 30 min prior to stimulation with PTN resulted in complete elimination of PTN-induced carboxy-DCF fluorescence elevation (Fig. 3A), similarly to its effect on PTN-induced cell migration (Polykratis et al., 2005). Since sodium orthovanadate is a non-specific tyrosine phosphatase inhibitor, we then specifically down-regulated the expression of RPTP $\beta / \zeta$ by siRNA, as previously described (Mikelis et al., 2009; Polykratis et al., 2005). Downregulation of RPTP $/ \zeta$ resulted in complete inhibition of PTN-induced carboxy-DCF fluorescence elevation (Fig. 3B), verifying involvement of $\mathrm{RPTP} / \zeta$ in this effect. Similarly to HUVEC, RPTP $\beta / \zeta$ seems to be involved in PTN-induced carboxy-DCF fluorescence elevation in LNCaP cells (Supplementary Figure S2).

Integrin $\alpha_{\nu} \beta_{3}$ is involved in PTN-induced elevation of carboxy-DCF fluorescence

We have previously shown that $\alpha_{\nu} \beta_{3}$ integrin is forming a cell surface functional complex with RPTP $\beta / \zeta$ and is also required for PTNinduced endothelial cell migration (Mikelis et al., 2009). Since we know that PTN interacts with the specificity loop of the $\beta_{3}$ integrin extracellular domain (Mikelis et al., 2009), we tested the effect of a synthetic peptide that corresponds to aminoacids ${ }^{177}$ CYDMKTTC $^{184}$ of the specificity loop (B3 peptide) and inhibits PTN-induced cell migration (Mikelis et al., 2009) on PTN-induced carboxy-DCF
A

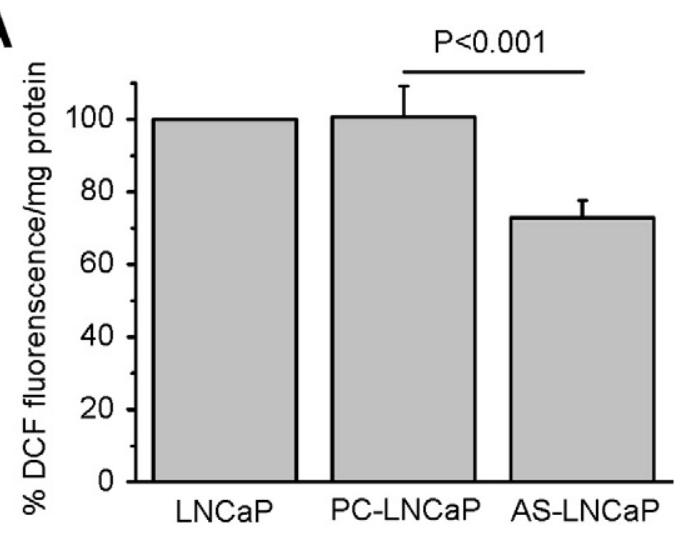

B

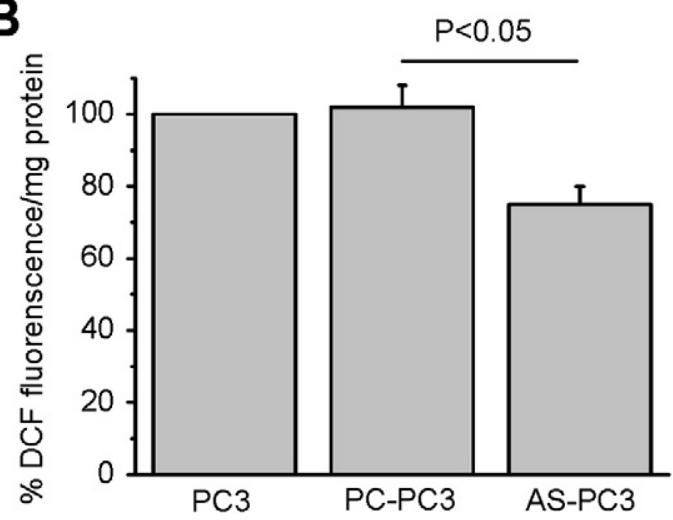

Fig. 2. Levels of endogenous PTN correlate with intracellular carboxy-DCF fluorescence LNCaP (A) and PC3 (B) cells with decreased expression of endogenous PTN were used to measure intracellular carboxy-DCF fluorescence. Results are expressed as mean \pm s.e.m. of the percent change of carboxy-DCF fluorescence per mg of total protein compared with the corresponding non-transfected cells (set as default $=100$ ). LNCaP, nontransfected LNCaP cells; PC-LNCaP, cells transfected with the plasmid containing only the neomycin resistance gene; AS-LNCaP, cells transfected with the plasmid containing antisense PTN; PC3, non-transfected PC3 cells; PC-PC3, cells transfected with the plasmid containing only the neomycin resistance gene; AS-PC3, cells transfected with the plasmid containing antisense PTN.

fluorescence elevation. Pre-treatment of HUVEC with B3 peptide prior to PTN stimulation completely abolished PTN-induced carboxy-DCF fluorescence elevation. A scrambled B3 peptide at the same concentration had no effect (Fig. 4A).

In order to further evaluate the role of $\alpha_{\nu} \beta_{3}$ on PTN-induced carboxy-DCF fluorescence elevation, we used mock-transfected $\mathrm{CHO}$ cells that express $\alpha_{v}$ but are negative for $\beta_{3}$, as well as CHO cells overexpressing wild-type $\beta_{3}$ or mutant $\beta_{3} \mathrm{Y} 773 \mathrm{~F} / \mathrm{Y} 785 \mathrm{~F}$ (Mikelis et al., 2009). PTN increased carboxy-DCF fluorescence in $\mathrm{CHO}$ cells expressing wild-type $\beta_{3}$, but slightly decreased carboxy-DCF fluorescence in $\mathrm{CHO}$ cells that do not express $\beta_{3}$ or express the mutant $\beta_{3}$ subunit (Fig. 4B), similarly to what has been previously observed for cell migration (Mikelis et al., 2009).

Finally, we tested the effect of PTN on carboxy-DCF fluorescence in human glioma M059K cells that do not express cell surface $\alpha_{\nu} \beta_{3}$ (Mikelis et al., 2009). PTN caused a slight decrease in carboxy-DCF fluorescence in these cells (Fig. 4C), similarly to its effect on cell migration (Mikelis et al., 2009).

PTN-induced elevation of carboxy-DCF fluorescence lays down-stream of c-src, PI3K and ERK1/2 activation

It is known that c-src, PI3K and ERK1/2 kinases participate in the PTN signaling pathway that involves RPTP $\beta / \zeta$ and $\alpha_{\nu} \beta_{3}$, and play a role in PTN-induced cell migration (Koutsioumpa et al., 2013; Mikelis et al., 
A

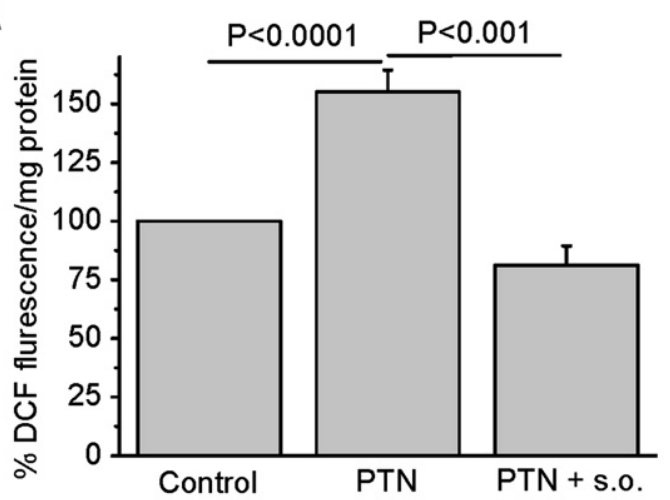

B

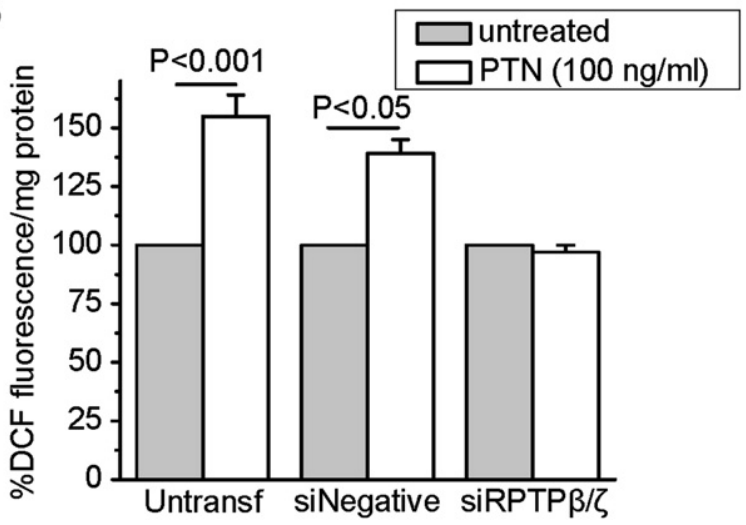

Fig. 3. RPTP $\beta / \zeta$ is involved in PTN-induced elevation of carboxy-DCF fluorescence. Serum starved HUVEC were pre-incubated with the tyrosine phosphatase inhibitor sodium orthovanadate (20 nM) (A) or siRNA for RPTP $\beta / \zeta$ (B) and then stimulated with PTN $(100 \mathrm{ng} / \mathrm{ml})$. Results are expressed as mean \pm s.e.m. of the percent change of carboxyDCF fluorescence per mg of total protein in PTN-stimulated cells compared with the corresponding untreated cells (set as default $=100$ ). s.o.: cells pre-treated with sodium orthovanadate; siRPTP $\beta / \zeta$ : cells transfected with siRNA for RPTP $\beta / \zeta$; siNegative: cells transfected with a negative control siRNA; untransf: untransfected cells.

2009; Polykratis et al., 2005). In the present study, we investigated a possible role of these signaling molecules in PTN-induced carboxyDCF fluorescence elevation. Pretreatment of HUVEC with the c-src inhibitor PP1, the PI3K inhibitor wortmannin and the ERK1/2 activation inhibitor U0126 at concentrations that inhibit PTN-induced cell migration (Mikelis et al., 2009; Polykratis et al., 2005), completely inhibited the effect of PTN on carboxy-DCF fluorescence (Fig. 5).

Xanthine oxidase mediates the PTN-induced elevation of carboxy-DCF fluorescence

Pre-treatment of HUVEC with catalase abolished PTN-induced carboxy-DCF fluorescence elevation, suggesting that hydrogen peroxide may be involved (Fig. 6A). To evaluate the enzymatic origin of ROS generation in response to PTN, we used the NADPH oxidase inhibitors AEBSF, apocynin and VAS2870, and the XO inhibitors allopurinol and febuxostat. Treatment of HUVEC with allopurinol or febuxostat prior to stimulation with PTN completely abolished PTN-induced carboxy-DCF fluorescence elevation, while the NADPH inhibitors gave differential effects. Apocynin completely abolished, while AEBSF and VAS2780 did not significantly affect PTN-induced carboxy-DCF fluorescence elevation (Fig. 6A). These data suggest that XO but not NADPH oxidase is involved in PTN-stimulated ROS production in HUVEC. Down-regulation of endogenous XO expression by siRNA abolished PTN-induced carboxy-DCF fluorescence elevation, verifying XO involvement (Fig. 6B).
We next studied whether PTN-induced endothelial cell migration was mediated by elevation of intracellular ROS levels. Pre-treatment of HUVEC with catalase inhibited PTN-induced HUVEC migration, suggesting that ROS are required for the stimulatory effect of PTN on cell migration. Pre-treatment of HUVEC with allopurinol and febuxostat also completely abolished PTN-induced HUVEC migration, suggesting that $\mathrm{XO}$ is involved in PTN-induced migration. The different NADPH inhibitors used gave differential effects. Apocynin completely abolished PTN-induced HUVEC migration. VAS2780 had a small inhibitory effect on HUVEC migration by itself, but did not inhibit the stimulatory effect of PTN. Finally, in the presence of AEBSF, PTN significantly decreased HUVEC migration (Fig. 6C). Down-regulation of endogenous XO expression by siRNA abolished PTN-induced endothelial cell migration, verifying XO involvement (Fig. 6D).

\section{Discussion}

Several previous studies have shown generation of ROS in a variety of cells upon stimulation by different growth factors and involvement of ROS in growth factor actions (Hatziapostolou et al., 2006; Lange et al., 2009; Polytarchou and Papadimitriou, 2004, 2005; Ushio-Fukai, 2007). In the present study, we show for the first time that PTN also increases ROS production in a concentration- and time-dependent manner in human endothelial and prostate cancer cells. Although carboxy-DCF fluorescence can be increased by several oxidants (Kalyanaraman et al., 2012), the observation that catalase abolished PTN-induced carboxy-DCFDA oxidation, suggests involvement of hydrogen peroxide. The enzyme responsible for PTN-induced ROS production seems to be XO, based on the observation that XO pharmacological inhibitors and down-regulation of endogenous XO abolished the stimulatory effect of PTN on ROS production. The lack of effect of the NADPH oxidase inhibitors AEBSF and VAS22780 on PTN-induced ROS production suggests that NADPH oxidase is not involved, while the inhibitory effect of apocynin could be due to its scavenging properties for hydrogen peroxide (Heumüller et al., 2008).

$\mathrm{XO}$ is a major source of ROS and has been linked to the pathogenesis of several inflammatory diseases, such as atherosclerosis (Nomura et al., 2014; Schröder et al., 2006), chronic obstructive pulmonary disease (Komaki et al., 2005), interstitial fibrosis in chronic kidney disease (Omori et al., 2012), inflammation in Type I diabetes (Romagnoli et al., 2010) and vascular inflammation (Sabán-Ruiz et al., 2013). PTN is also involved in inflammatory pathologies (Pantazaka and Papadimitriou, 2012; Papadimitriou et al., 2009; Silver et al., 2012; Yokoi et al., 2012; Zhang et al., 2013) and activation of XO might be one of the mechanisms of its pro-inflammatory effects. In favor of this notion are data showing that interferon gamma (IFN $\gamma$ ), which is a known inducer of XO (Dupont et al., 1992), up-regulates PTN expression in capillaries of human atherosclerotic plaques through JAKs/STATs downstream effectors (Li et al., 2010). Since XO is involved in the pathogenesis of atherosclerosis (Nomura et al., 2014) and its activation is also mediated by JAKs/STATs (Wang et al., 2008), one can speculate that PTN mediates the stimulatory effect of IFN $\gamma$ on XO activation, similarly to its role as a mediator of the stimulatory effects of hydrogen peroxide (Polytarchou et al., 2005), nitric oxide (Polytarchou et al., 2009) and fibroblast growth factor-2 (Hatziapostolou et al., 2006). In the same line, hypoxia stimulates both PTN expression (Antoine et al., 2005) and XO activation (Wang et al., 2008), suggesting that hypoxia-induced XO activation might be also mediated by PTN over-expression.

Involvement of ROS in the stimulatory effects of growth factors has been previously shown, with NADPH oxidase considered as the main source of ROS (Sies, 2014). There is also one study showing involvement of endogenous XO in VEGF-induced actions, such as endothelial cell survival (Kou et al., 2008), suggesting that multiple sources of ROS may orchestrate the various effects of growth factors. In the present study we show that XO mediates the stimulatory effect of PTN on cell migration and could be hypothesized that XO might also mediate the 
A

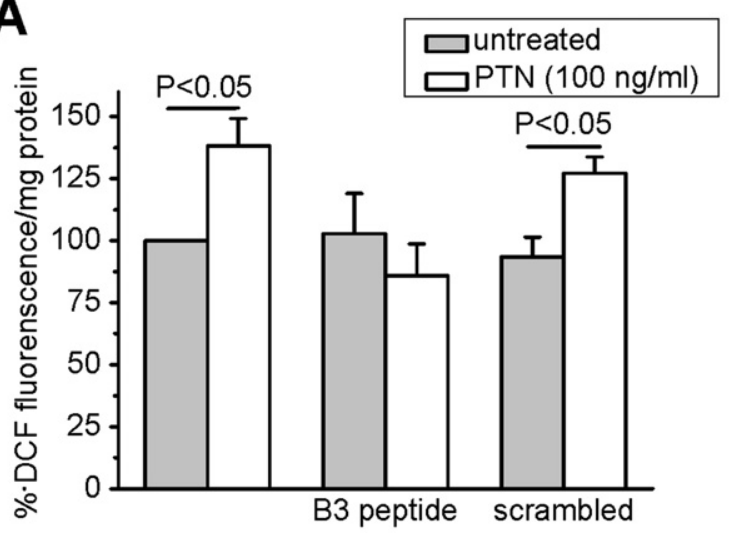

B

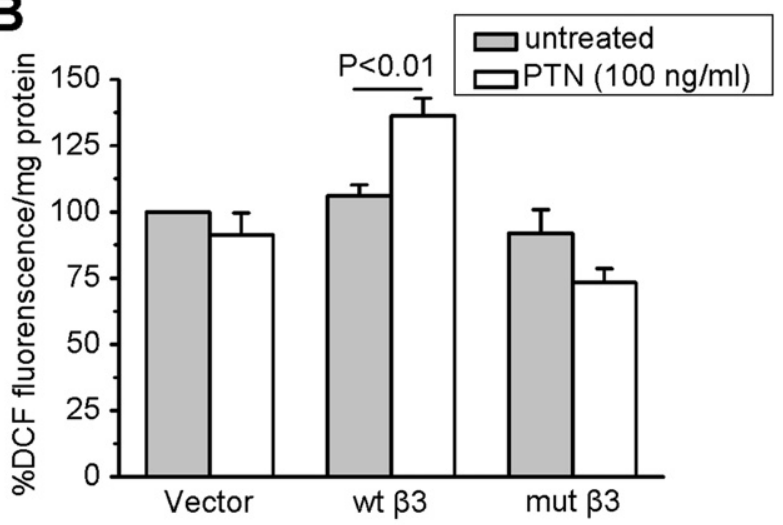

C

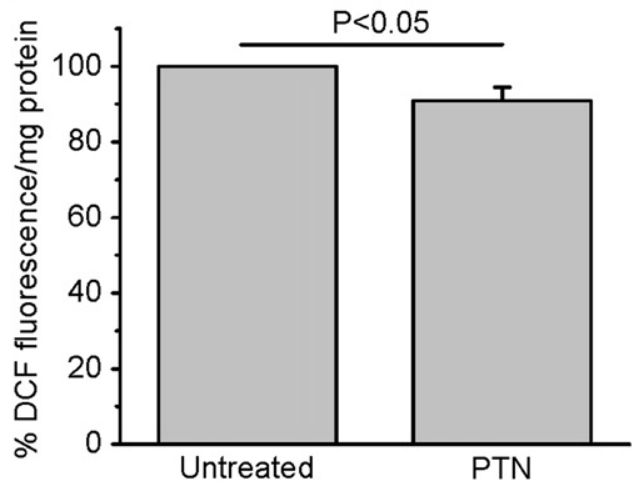

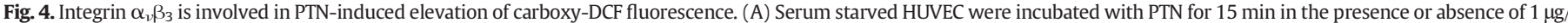

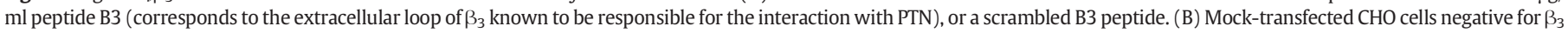

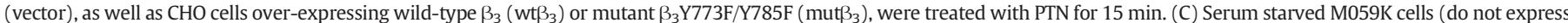

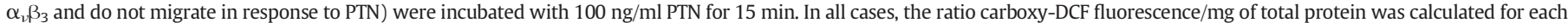

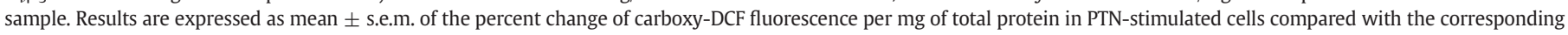
untreated cells ( set as default $=100$ ).

anti-apoptotic effect of PTN. This notion is in line with our data that human prostate cancer cells that express low PTN levels have increased rates of apoptosis (Tsirmoula et al., 2012), decreased migration (Hatziapostolou et al., 2005; Tsirmoula et al., 2012) and decreased

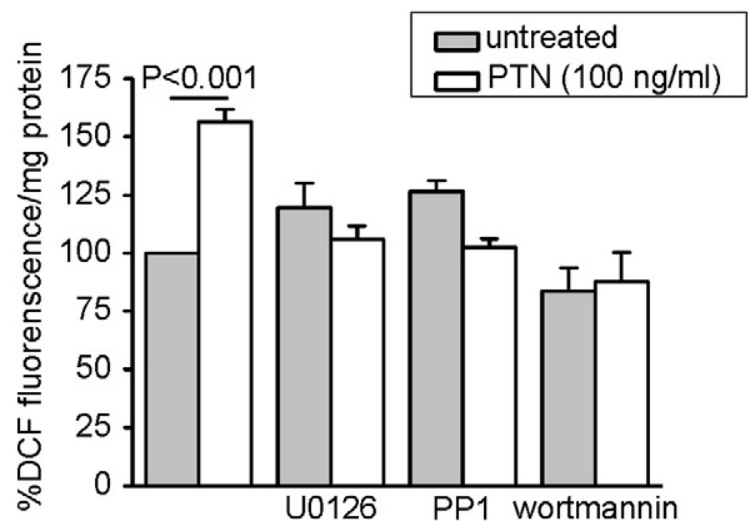

Fig. 5. PTN-induced carboxy-DCF fluorescence elevation was inhibited by inhibition of csrc and PI3-K or inhibition of ERK1/2 activation. Serum starved HUVEC were pre-incubated for 30 min with the c-src inhibitor PP1 $(20 \mathrm{nM})$, the PI3K inhibitor wortmannin $(100 \mathrm{nM})$ or the ERK1/2 activation inhibitor U0126 (20 nM) and then stimulated with PTN for $15 \mathrm{~min}$. The ratio carboxy-DCF fluorescence $/ \mathrm{mg}$ of total protein was calculated for each sample. Results are expressed as mean \pm s.e.m. of the percent change of carboxy-DCF fluorescence per mg of total protein in PTN-stimulated cells compared with the untreated cells ( set as default $=100$ ). growth and metastases in vivo (Tsirmoula et al., 2012), and based on the present study, these same cells have lower levels of ROS compared with the corresponding cells expressing normal PTN levels. These data are also in line with data showing that XO levels are elevated in patients with prostate cancer (Samra et al., 2011), as well as several other types of cancer, such as breast, lung and bladder cancer, non-Hodgkin lymphoma and acute lymphoblastic lymphoma (Samra et al., 2011; Güleç et al., 2003). On the other hand, decreased XO activity has been found in hepatomas (Prajda and Weber, 1975; Prajda et al., 1976) and gastric cancer (Linder et al., 2006), which could be explained by the hypothesis that XO expression may be cancer type-specific. XO has been also shown to stabilize hypoxia-inducible factor-1alpha (HIF-1 $\alpha$ ) under hypoxic conditions in glioma cells (Griguer et al., 2006), which is also in favor of a regulatory role of XO in cancer and angiogenesis. Hydrogen peroxide activates HIF- $1 \alpha$ in cancer and/or stroma cells, contributing to cancer metabolism and metastasis (Lisanti et al., 2011), although up to date the enzymatic sources of cancer-associated hydrogen peroxide remain poorly studied. Taking into account that $\mathrm{XO}$ has been linked to several inflammatory conditions, as discussed above, it would be interesting to further investigate its role in cancer.

By using several pharmacological and genetic approaches, we found that the cell surface functional complex of RPTP $\beta / \zeta / \alpha_{v} \beta_{3}$ that is required for PTN-induced cell migration (Mikelis et al., 2009), is also involved in PTN-induced ROS production. Moreover, the enzymatic source of ROS lays down-stream of c-Src, PI3K and ERK1/2, which are also involved in PTN-induced cell migration downstream of RPTP $\beta / \zeta$ (Polykratis et al., 2005). Although ROS are known to act as growth factor downstream signaling molecules that inhibit protein tyrosine 

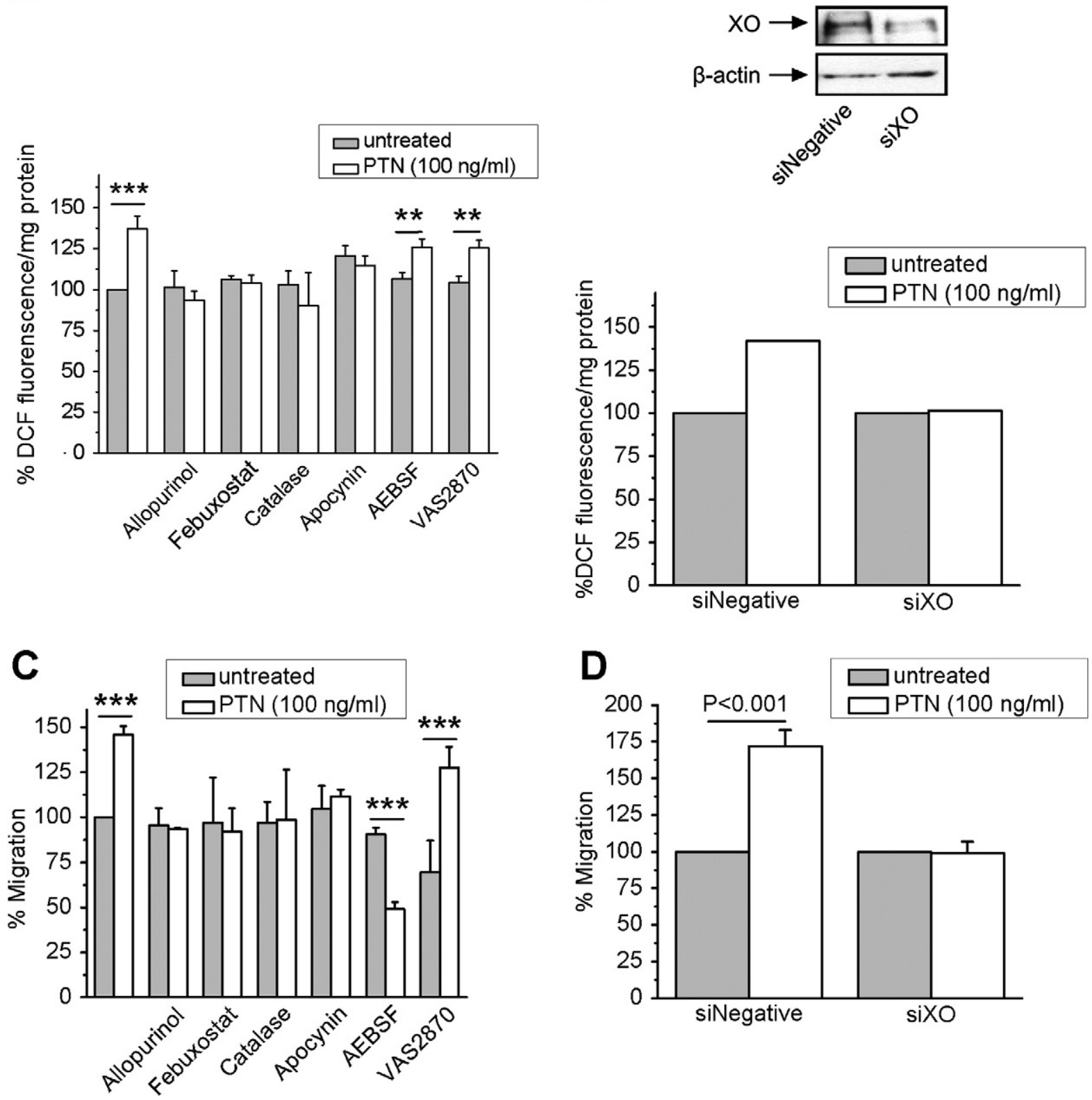

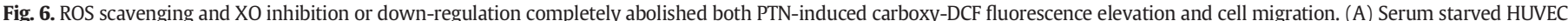

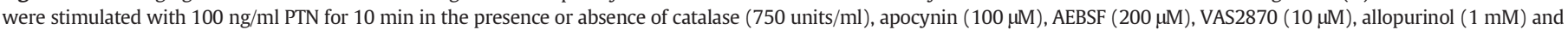

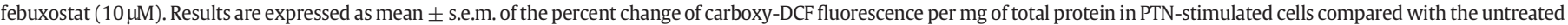

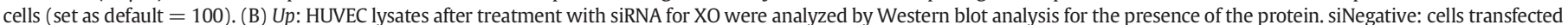

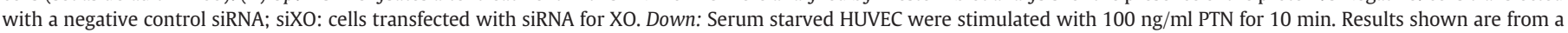

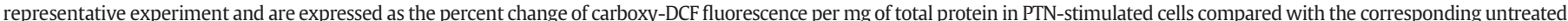

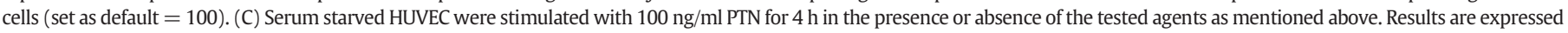

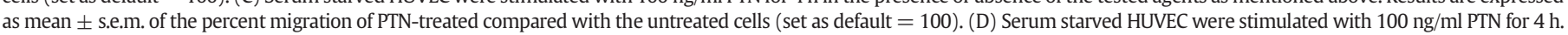

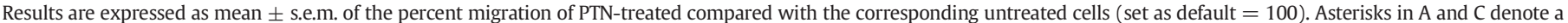
statistically significant difference. ${ }^{* *} P<0.01,{ }^{* * *} P<0.001$.

phosphatases (Ray et al., 2012) and are produced in response to integrin activation either independently or in cooperation with growth factor receptors (Goitre et al., 2012), to the best of our knowledge this is the first study that shows implication of a receptor tyrosine phosphatase, RPTP $\beta / \zeta$, on ROS production. Moreover, although not many data are available on the signaling molecules affecting XO activation in endothelial or other types of cells, ERK1/2 have been previously shown to lay upstream and to induce XO enzymatic activity in response to mechanical stress in rat pulmonary microvascular endothelial cells (Abdulnour et al., 2006), in line with our observation.

Increased ROS levels as a result of PTN stimulation of endothelial cells correlate with increased cell migration. Similarly, higher PTN expression correlates with higher endogenous ROS levels (this study) and increased migration (Hatziapostolou et al., 2005; Tsirmoula et al., 2012 ) in human prostate cancer cells. The role of XO in PTN-induced endothelial cell migration is supported by the data showing that allopurinol and febuxostat, similarly to ROS scavenging and down-regulation 
of endogenous XO expression, completely abolished PTN-induced cell migration. The NADPH inhibitors gave conflicting results: apocynin caused complete inhibition of cell migration, while VAS2780 had no effect, similarly to their corresponding effects on ROS levels. On the other hand, AEBSF not only inhibited the stimulatory effect of PTN, but in its presence, PTN significantly inhibited cell migration. Besides inhibiting NADPH oxidase, AEBSF can also act as a serine protease inhibitor (Wind et al., 2010). It is not known whether this is the reason for the decrease in cell migration caused by PTN in the presence of AEBSF, but it may be a possibility since PTN has been shown to enhance plasminogen activator activity in bovine aortic endothelial cells (Kojima et al., 1995). Considering the basic role of plasmin in endothelial cell migration (Heissig et al., 2012), it could be hypothesized that PTN exerts its stimulatory effect on cell migration by activating serine proteases.

\section{Conclusions}

XO-mediated ROS production through the cell membrane functional complex of RPTP $\beta / \zeta$ and $\alpha_{\nu} \beta_{3}$ and activation of c-src, PI3-K and ERK1/2 kinases, is required for PTN-induced cell migration. Further studies are in progress to elucidate the role of XO and ROS in PTN signaling and functions in endothelial and other types of cells.

\section{Acknowledgments}

This research has been co-financed by the European Union (European Social Fund-ESF) and Greek national funds through the Operational Program "Education and Lifelong Learning" of the National Strategic Reference Framework (NSRF)-Research Funding Program: Heracleitus II (MIS: 346791). Investing in knowledge society through the European Social Fund.

\section{Appendix A. Supplementary data}

Supplementary data to this article can be found online at http://dx. doi.org/10.1016/j.mvr.2015.01.001.

\section{References}

Abdulnour, R.E., Peng, X., Finigan, J.H., Han, E.J., Hasan, E.J., Birukov, K.G., Reddy, S.P., Watkins III, J.E., Kayyali, U.S., Garcia, J.G., Tuder, R.M., Hassoun, P.M., 2006. Mechanical stress activates xanthine oxidoreductase through MAP kinase-dependent pathways. Am. J. Physiol. Lung Cell. Mol. Physiol. 291, L345-L353.

Antoine, M., Tag, C.G., Wirz, W., Borkham-Kamphorst, E., Sawitza, I., Gressner, A.M., Kiefer P., 2005. Upregulation of pleiotrophin expression in rat hepatic stellate cells by PDGF and hypoxia: implications for its role in experimental biliary liver fibrogenesis. Biochem. Biophys. Res. Commun. 337, 1153-1164.

Bir, S.C., Kolluru, G.K., Fang, K., Kevil, C.G., 2012. Redox balance dynamically regulates vascular growth and remodeling. Semin. Cell Dev. Biol. 23, 745-757.

Bryan, N., Ahswin, H., Smart, N., Bayon, Y., Wohlert, S., Hunt, J.A., 2012. Reactive oxygen species (ROS) - a family of fate deciding molecules pivotal in constructive inflammation and wound healing. Eur. Cell Mater. 24, 249-265.

Dhar-Mascareno, M., Chen, J., Zhang, R.H., Carcamo, J.M., Golde, D.W., 2003. Granulocytemacrophage colony-stimulating factor signals for increased glucose transport via phosphatidylinositol 3-kinase- and hydrogen peroxide-dependent mechanisms. J. Biol. Chem. 278, 11107-11114.

Dupont, G.P., Huecksteadt, T.P., Marshall, B.C., Ryan, U.S., Michael, J.R., Hoidal, J.R., 1992. Regulation of xanthine dehydrogenase and xanthine oxidase activity and gene expression in cultured rat pulmonary endothelial cells. J. Clin. Invest. 89, 197-202.

Goitre, L., Pergolizzi, B., Ferro, E., Trabalzini, L., Retta, S.F., 2012. Molecular crosstalk between integrins and cadherins: do reactive oxygen species set the talk? J. Signal Transduct. 2012, 807682

Griguer, C.E., Oliva, C.R., Kelley, E.E., Giles, G.I., Lancaster Jr., J.R., Gillespie, G.Y., 2006 Xanthine oxidase-dependent regulation of hypoxia-inducible factor in cancer cells. Cancer Res. 66, 2257-2263.

Gülec, M., Akin, H., Yüce, H.H., Ergin, E., Elyas, H., Yalçin, O., Akyol, O., 2003. Adenosine deaminase and xanthine oxidase activities in bladder washing fluid from patients with bladder cancer: a preliminary study. Clin. Biochem. 36, 193-196.

Hatziapostolou, M., Delbe, J., Katsoris, P., Polytarchou, C., Courty, J., Papadimitriou, E., 2005. Heparin affin regulatory peptide is a key player in prostate cancer cell growth and angiogenicity. Prostate 65, 151-158.

Hatziapostolou, M., Polytarchou, C., Katsoris, P., Courty, J., Papadimitriou, E., 2006. Heparin affin regulatory peptide/pleiotrophin mediates fibroblast growth factor 2 stimulatory effects on human prostate cancer cells. J. Biol. Chem. 281, 32217-32226.
Heissig, B., Ohki-Koizumi, M., Tashiro, Y., Gritli, I., Sato-Kusubata, K., Hattori, K., 2012. New functions of the fibrinolytic system in bone marrow cell-derived angiogenesis. Int. J. Hematol. 95, 131-137.

Heumüller, S., Wind, S., Barbosa-Sicard, E., Schmidt, H.H., Busse, R., Schröder, K., Brandes, R.P., 2008. Apocynin is not an inhibitor of vascular NADPH oxidases but an antioxidant. Hypertension 51, 211-217.

Holmström, K.M., Finkel, T., 2014. Cellular mechanisms and physiological consequences of redox-dependent signalling. Nat. Rev. Mol. Cell Biol. 15, 411-421.

Kalyanaraman, B., Darley-Usmar, V., Davies, K.J., Dennery, P.A., Forman, H.J., Grisham, M.B., Mann, G.E., Moore, K., Roberts 2nd, L.J., Ischiropoulos, H., 2012. Measuring reactive oxygen and nitrogen species with fluorescent probes: challenges and limitations. Free Radic. Biol. Med. 52, 1-6.

Kim, Y.W., Byzova, T.V., 2014. Oxidative stress in angiogenesis and vascular disease. Blood $123,625-631$.

Kojima, S., Inui, T., Muramatsu, H., Kimura, T., Sakakibara, S., Muramatsu, T., 1995. Midkine is a heat and acid stable polypeptide capable of enhancing plasminogen activator activity and neurite outgrowth extension. Biochem. Biophys. Res. Commun. 216, 574-581.

Komaki, Y., Sugiura, H., Koarai, A., Tomaki, M., Ogawa, H., Akita, T., Hattori, T., Ichinose, M., 2005. Cytokine-mediated xanthine oxidase upregulation in chronic obstructive pulmonary disease's airways. Pulm. Pharmacol. Ther. 18, 297-302.

Kou, B., Ni, J., Vatish, M., Singer, D.R., 2008. Xanthine oxidase interaction with vascular endothelial growth factor in human endothelial cell angiogenesis. Microcirculation 15, 251-267.

Koutsioumpa, M., Polytarchou, C., Courty, J., Zhang, Y., Kieffer, N., Mikelis, C., Skandalis S.S., Hellman, U., Iliopoulos, D., Papadimitriou, E., 2013. Interplay between $\alpha v \beta 3$ integrin and nucleolin regulates human endothelial and glioma cell migration. J. Biol. Chem. 288, 343-354.

Lange, S., Heger, J., Euler, G., Wartenberg, M., Piper, H.M., Sauer, H., 2009. Platelet-derived growth factor BB stimulates vasculogenesis of embryonic stem cell-derived endothelial cells by calcium-mediated generation of reactive oxygen species. Cardiovasc. Res. 81, 159-168.

Li, F., Tian, F., Wang, L., Williamson, I.K., Sharifi, B.G., Shah, P.K., 2010. Pleiotrophin (PTN) is expressed in vascularized human atherosclerotic plaques: IFN-\{gamma\}/JAK/ STAT1 signaling is critical for the expression of PTN in macrophages. FASEB J. 24 $810-822$.

Linder, N., Haglund, C., Lundin, M., Nordling S, Ristimäki, A, Kokkola, A., Mrena, J., Wiksten, J.P., Lundin, J., 2006. Decreased xanthine oxidoreductase is a predictor of poor prognosis in early-stage gastric cancer. J. Clin. Pathol. 59, 965-971.

Lisanti, M.P., Martinez-Outschoorn, U.E., Lin, Z., Pavlides, S., Whitaker-Menezes, D., Pestell, R.G., Howell, A., Sotgia, F., 2011. Hydrogen peroxide fuels aging, inflammation, cancer metabolism and metastasis: the seed and soil also needs "fertilizer". Cell Cycle 10, 2440-2449.

Lu, K.V., Jong, K.A., Kim, G.Y., Singh, J., Dia, E.Q., Yoshimoto, K., Wang, M.Y., Cloughesy, T.F., Nelson, S.F., Mischel, P.S., 2005. Differential induction of glioblastoma migration and growth by two forms of pleiotrophin. J. Biol. Chem. 280, 26953-26964.

Mikelis, C., Sfaelou, E., Koutsioumpa, M., Kieffer, N., Papadimitriou, E., 2009. Integrin alpha(v)beta(3) is a pleiotrophin receptor required for pleiotrophin-induced endothelial cell migration through receptor protein tyrosine phosphatase beta/zeta. FASEB J. 23, 1459-1469.

Naik, E., Dixit, V.M., 2011. Mitochondrial reactive oxygen species drive proinflammatory cytokine production. J. Exp. Med. 208, 417-420.

Nomura, J., Busso, N., Ives, A., Matsui, C., Tsujimoto, S., Shirakura, T., Tamura, M. Kobayashi, T., So, A., Yamanaka, Y., 2014. Xanthine oxidase inhibition by febuxostat attenuates experimental atherosclerosis in mice. Sci. Rep. 4, 4554

Omori, H., Kawada, N., Inoue, K., Ueda, Y., Yamamoto, R., Matsui, I., Kaimori, J., Takabatake, Y., Moriyama, T., Isaka, Y., Rakugi, H., 2012. Use of xanthine oxidase inhibitor febuxostat inhibits renal interstitial inflammation and fibrosis in unilateral uretera obstructive nephropathy. Clin. Exp. Nephrol. 16, 549-556.

Pantazaka, E., Papadimitriou, E., 2012. PTN (pleiotrophin). Atlas Genet. Cytogenet. Oncol Haematol. 16, 821-837.

Papadimitriou, E., Mikelis, C., Lampropoulou, E., Koutsioumpa, M., Theochari, K., Tsirmoula, S., Theodoropoulou, C., Lamprou, M., Sfaelou, E., Vourtsis, D., Boudouris, P., 2009. Roles of pleiotrophin in tumor growth and angiogenesis. Eur. Cytokine Netw. 20, 180-190.

Polykratis, A., Katsoris, P., Courty, J., Papadimitriou, E., 2005. Characterization of heparin affin regulatory peptide signaling in human endothelial cells. J. Biol. Chem. 280, 22454-22461

Polytarchou, C., Papadimitriou, E., 2004. Antioxidants inhibit angiogenesis in vivo through down-regulation of nitric oxide synthase expression and activity. Free Radic. Res. 38 501-508.

Polytarchou, C., Papadimitriou, E., 2005. Antioxidants inhibit human endothelial cell functions through down-regulation of endothelial nitric oxide synthase activity. Eur. J. Pharmacol. 510, 31-38.

Polytarchou, C., Hatziapostolou, M., Papadimitriou, E., 2005. Hydrogen peroxide stimulates proliferation and migration of human prostate cancer cells through activation of activator protein-1 and up-regulation of the heparin affin regulatory peptide gene. J. Biol. Chem. 280, 40428-40435

Polytarchou, C., Hatziapostolou, M., Poimenidi, E., Mikelis, C., Papadopoulou, A., Parthymou, A., Papadimitriou, E., 2009. Nitric oxide stimulates migration of human endothelial and prostate cancer cells through up-regulation of pleiotrophin expression and its receptor protein tyrosine phosphatase beta/zeta. Int. J. Cancer $124,1785-1793$

Prajda, N., Weber, G., 1975. Malignant transformation-linked imbalance: decreased xanthine oxidase activity in hepatomas. FEBS Lett. 59, 245-249.

Prajda, N., Morris, H.P., Weber, G., 1976. Imbalance of purine metabolism in hepatomas of different growth rates as expressed in behavior of xanthine oxidase (EC 1.2.3.2). Cancer Res 36, 4639-4646. 
Ray, P.D., Huang, B.W., Tsuji, Y., 2012. Reactive oxygen species (ROS) homeostasis and redox regulation in cellular signaling. Cell. Signal. 24, 981-990.

Romagnoli, M., Gomez-Cabrera, M.C., Perrelli, M.G., Biasi, F., Pallardó, F.V., Sastre, J., Poli, G., Viña, J., 2010. Xanthine oxidase-induced oxidative stress causes activation of NFkappaB and inflammation in the liver of type I diabetic rats. Free Radic. Biol. Med. 49, 171-177.

Sabán-Ruiz, J., Alonso-Pacho, A., Fabregate-Fuente, M., de la Puerta González-Quevedo, C., 2013. Xanthine oxidase inhibitor febuxostat as a novel agent postulated to act against vascular inflammation. Antiinflamm. Antiallergy Agents Med. Chem. 12, 94-99.

Samra, Z.Q., Pervaiz, S., Shaheen, S., Dar, N., Athar, M.A., 2011. Determination of oxygen derived free radicals producer (xanthine oxidase) and scavenger (paraoxonase1) enzymes and lipid parameters in different cancer patients. Clin. Lab. 57, 741-747.

Schröder, K., Vecchione, C., Jung, O., Schreiber, J.G., Shiri-Sverdlov, R., van Gorp, P.J., Busse, R., Brandes, R.P., 2006. Xanthine oxidase inhibitor tungsten prevents the development of atherosclerosis in ApoE knockout mice fed a Western-type diet. Free Radic. Biol. Med. 41, 1353-1360.

Sies, $\mathrm{H}$., 2014. Role of metabolic $\mathrm{H}_{2} \mathrm{O}_{2}$ generation: redox signaling and oxidative stress J. Biol. Chem. 289, 8735-8741.

Silver, K., Desormaux, A., Freeman, L.C., Lillich, J.D., 2012. Expression of pleiotrophin, an important regulator of cell migration, is inhibited in intestinal epithelial cells by treatment with non-steroidal anti-inflammatory drugs. Growth Factors 30, 258-266.

Terman, B.I., Dougher-Vermazen, M., Carrion, M.E., Dimitrov, D., Armellino, D.C. Gospodarowicz, D., Bohlen, P., 1992. Identification of the KDR tyrosine kinase as a receptor for vascular endothelial cell growth factor. Biochem. Biophys. Res. Commun. 187, 1579-1586.

Tsirmoula, S., Dimas, K., Hatziapostolou, M., Lamprou, M., Ravazoula, P., Papadimitriou, E., 2012. Implications of pleiotrophin in human PC3 prostate cancer cell growth in vivo. Cancer Sci. 103, 1826-1832.

Ushio-Fukai, M., 2007. VEGF signaling through NADPH oxidase-derived ROS. Antioxid. Redox Signal. 9, 731-739.

Wang G. Oian, P. Jackson, F. R. Qian, G, Wu, G, 2008. Sequential activation of JAKs, STATs and xanthine dehydrogenase/oxidase by hypoxia in lung microvascular endothelial cells. Int. J. Biochem. Cell Biol. 40, 461-470.

Wilkinson-Berka, J.L., Rana, I., Armani, R., Agrotis, A., 2013. Reactive oxygen species, Nox and angiotensin II in angiogenesis: implications for retinopathy. Clin. Sci. (Lond.) 124, 597-615.

Wind, S., Beuerlein, K., Eucker, T., Müller, H., Scheurer, P., Armitage, M.E., Ho, H., Schmidt, H.H., Wingler, K., 2010. Comparative pharmacology of chemically distinct NADPH oxidase inhibitors. Br. J. Pharmacol. 161, 885-898.

Yokoi, H., Kasahara, M., Mori, K., Ogawa, Y., Kuwabara, T., Imamaki, H., Kawanishi, T., Koga, K., Ishii, A., Kato, Y., Mori, K.P., Toda, N., Ohno, S., Muramatsu, H., Muramatsu, T., Sugawara, A., Mukoyama, M., Nakao, K., 2012. Pleiotrophin triggers inflammation and increased peritoneal permeability leading to peritoneal fibrosis. Kidney Int. 81, $160-169$.

Zhang, O., Tao, K., Huang, W., Tian, Y., Liu, X., 2013. Elevated expression of pleiotrophin in human hypertrophic scars. J. Mol. Histol. 44, 91-96. 